\title{
Decoupled Fuzzy Sliding Mode Control for a Synchronous Motor Speed Control
}

\author{
Mohamed M. Abdelsalam \\ Assist. Lecturer \\ Mansoura University, Egypt
}

\author{
Marwa F. Areed \\ Ph.d., Lecturer \\ Mansoura University, Egypt
}

\begin{abstract}
This paper introduces a new technique for controlling the speed of Permanent Magnet Synchronous Motor (PMSM). This technique depends on two well known control methods; the first control method is known the variable structure system (VSS), which has a main advantage known as an sliding mode property. The second method depends on fuzzy logic control. A combination between the two mentioned above methods is suggested in this paper. In addition for simplifying the suggested technique, the model of PMSM is decoupled. Simulation results illustrate the validity and the effectiveness of the new suggested technique.
\end{abstract}

\section{Keywords}

Decoupled, Fuzzy Sliding Mode Control, Permanent Magnet Synchronous Motor, Pulse Width Modulation.

\section{INTRODUCTION}

Variable structure systems (VSS) are a control theory appeared in the second half of the last century. The main phenomenon in VSS is what so called Sliding mode [1-3]. The VSS is mainly a discontinuous control technique. If a sliding surface is properly designed, then the representative point will be forced by the control vector to hit the sliding surface. The discontinuous control forces the representative point to slide on the chosen sliding surface and reaches the origin (see Fig. 1), Around the surface s, is often irritated by high frequency oscillations known as chattering [4-5].

Sliding mode control (SMC) can exponentially drive the system state to a chattering sliding mode but tends to produce conservative designs [6-8]. Reduced chattering may be achieved without sacrificing robust performance by combining the attractive features of fuzzy logic with SMC [910]. Fuzzy Logic Control is a non-conventional and robust control law. It is suitable for nonlinear or complex systems characterized by parametric fluctuation or uncertainties. The advantages of the FSMC are that it is not directly related to a mathematical model of the controlled systems as the SMC, assures performance and stability and reduces the number of fuzzy rules [11-13].

In this paper, a decoupled fuzzy sliding-mode control (DFSMC) design strategy is used to control the speed of PMSM. The motor system is divided into two subsystems with different switching surfaces to achieve the desired speed.

\section{MATHEMATICAL MODE OF PMSM}

PMSMs are increasing applied in several areas such as traction, automobiles, robotics and aerospace technology. The power density of PMSM is higher than one of induction motor with the same ratings due to the no stator power dedicated to the magnetic field production. Nowadays, PMSM is designed not only to be more powerful but also with lower mass and lower moment of inertia. PMSMs present numerous advantages like high efficiency, high torque to inertia ratio, high power density, reliability and long life [14-16].

Different topologies of PM machines are available e.g. radial flux machines, axial flux machines and transversal flux machines. The transversal flux machine is a relatively recent developed machine type particularly suited for direct drive i.e. high torque and relatively low speed. Axial flux machines have been used in the both low speed direct drive and high speed flywheel applications. Radial flux machines have been also considered for hybrid electric vehicle [17-19].

For control unit design the synchronous motor is modeled in rotating rotor coordinates $\{\mathrm{d}, \mathrm{q}\}$. The PMSM model is given by the following differential equations as [20]:

$$
\begin{aligned}
& L \dot{i}_{d}=-R i_{d}+p \omega L_{d f} i_{d q}+u_{d t} \\
& L \dot{i}_{q}=-R i_{q}-p \omega L_{d} i_{d t}-\lambda p \omega+u_{q} \\
& T_{e}=1.5 p\left[\lambda i_{q}+\left(\dot{L}_{d}-L_{q}\right) \dot{i}_{d t} i_{q u}\right] \\
& J \dot{\omega}=T_{e}-\mu \omega+T_{L} \\
& \dot{\theta}=\omega
\end{aligned}
$$

Where

$\mathrm{u}_{\mathrm{d}}, \mathrm{u}_{\mathrm{q}}$ : The rotor voltages in $\{\mathrm{d}, \mathrm{q}\}$ coordinates $(\mathrm{V})$,

$\mathrm{i}_{\mathrm{d}}, \mathrm{iq}$ : rotor currents in $\{\mathrm{d}, \mathrm{q}\}$ coordinates $(\mathrm{A})$,

$\theta$ : The electrical rotor position ( $\mathrm{rad})$

$\omega$ : The angular velocity of the motor shaft in electrical . $(\mathrm{rad} / \mathrm{s})$

$\mathrm{Rd}, \mathrm{Rq}$ : The winding resistance of $\mathrm{d}$ and

$\mathrm{q}$ axis $(\Omega)$

$\mathrm{Ld}, \mathrm{Lq}$ : The inductance of $\mathrm{d}$ and $\mathrm{q}$ axis

$(\mathrm{H})$,

$\lambda$ : Rotor magnet flux linkage $(\mathrm{Wb})$

J : The rotor and shaft inertia (kg.m2)

$\mu$ : The coefficient of friction (N.m.s),

$\mathrm{p}$ : The number of permanent magnet

pole pairs, 
$\mathrm{T}_{\mathrm{L}}$ : The disturbing external torque

(N.m).

$\mathrm{T}_{\mathrm{e}}$ : The motor torque (N.m).

For $\mathrm{L}_{\mathrm{d}}=\mathrm{L}_{\mathrm{q}}=\mathrm{L}$ then the motor torque will be

$$
T_{i}=1.5 p \lambda i_{q}=K i_{q}
$$

Where : $\mathrm{k}$ is the motor torque constant

Substitution in Eq.(1),so the model can be written as:

$$
\begin{aligned}
& \dot{L i_{d}}=-R i_{d}+p \omega L i_{q}+u_{d} \\
& \dot{L} \dot{i}_{4}=-R i_{q}-p \omega L i_{d}-\frac{2}{3} k \omega+u_{q} \\
& J \dot{\omega}=T_{e}-\mu \omega+T_{L} \\
& \dot{\theta}=\omega
\end{aligned}
$$

\section{REPRESENTATION OF PMSM IN STATE SPACE MODEL.}

Let

$$
\begin{aligned}
& x_{1}=i_{d} \\
& x_{2}=i_{Q} \\
& x_{3}=\omega
\end{aligned}
$$

then the model can be written as

$$
\begin{aligned}
& \dot{x}_{1}=-\frac{R}{L} x_{1}+p x_{3} x_{2}+\frac{1}{L} u_{d_{1}} \\
& \dot{x}_{2}=-\frac{R}{L} x_{2}-p x_{3} x_{1}-\frac{2}{3 L} k x_{3}+\frac{1}{L} u_{q}
\end{aligned}
$$

So the model can be written as:

$$
\left[\begin{array}{l}
\dot{i_{d}} \\
\dot{i_{4}} \\
\dot{\theta}
\end{array}\right]\left[\begin{array}{l}
\dot{x}_{1} \\
\dot{x}_{2} \\
\dot{x}_{3}
\end{array}\right]=\left[\begin{array}{ccc}
-\frac{R}{L} & p x_{3} & 0 \\
-p x_{3} & -\frac{R}{L} & -\frac{2 K}{3 L} \\
0 & \frac{K}{J} & -\frac{\mu}{J}
\end{array}\right]\left[\begin{array}{c}
x_{1} \\
x_{2} \\
x_{3}
\end{array}\right]+\left[\begin{array}{cc}
\frac{1}{L} & 0 \\
0 & \frac{1}{L} \\
0 & 0
\end{array}\right]\left[\begin{array}{l}
u_{d} \\
u_{u}
\end{array}\right]-\left[\begin{array}{l}
0 \\
0 \\
0 \\
\frac{1}{J}
\end{array}\right] T_{L}
$$

From $(5,6)$, it is obvious that the dynamic model of PMSM is highly nonlinear because of the coupling between the speed and the electrical currents. In addition to the saturation effect of the magnetic circuit and the existing viscous friction.

The PMSM parameters are:

1.1 KW, 3000RPM, R=2.875 $\Omega, \mathrm{L}_{\mathrm{d}}=\mathrm{L}_{\mathrm{q}}=8.5 \mathrm{mH}, \mathrm{P}=4$ pair of

Poles( 8 poles $), \mathrm{J}=0.8 \times 10^{-3} \mathrm{Kg} \cdot \mathrm{m}^{2}, \mu=1$ N.m.s./rad.

\section{DECOUPLING AND SLIDING MODE CONTROL}

There is currently a large interest in sliding mode control algorithms due to their robustness properties and possibilities to decouple a high dimensional design problem into a set of lower dimensional independent sub-problems.

The switching surface: Consider a general type of system represented by the state equation,

$$
\dot{x}=f(x, u, t)
$$

The control $\mathrm{u}(\mathrm{x}, \mathrm{t})$ with its respective entry $\mathrm{u}_{\mathrm{i}}(\mathrm{x}, \mathrm{t})$ has the form

$$
\begin{gathered}
u_{i}(x, t)=\left\{\begin{array}{lll}
u_{i}^{+}(x, t) & \text { if } & s_{i}(x)>0 \\
u_{i}^{-}(x, t) & \text { if } & s_{i}(x)<0
\end{array}\right. \\
u_{i}^{-}(x, t) \quad u_{i}^{+}(x, t)
\end{gathered}
$$

Where are discontinuous functions. Since $u_{i}(x, t)$ undergoes discontinuity on the surface $s_{i}(x)=$ $0, s_{i}(x)=0$ is called a switching surface or switching hyperplane as in Fig.1

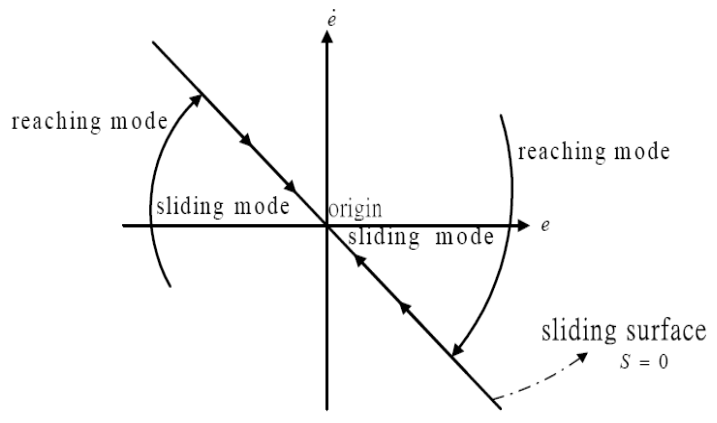

Fig.1: The Sliding surface (S)

With Sliding Condition

$$
\lim _{s \rightarrow 0^{+}} \dot{s}<0, \quad \lim _{s \rightarrow 0^{-}} \dot{s}>0
$$

Since it is required to produce a control input $u_{i}(x, t)$ that applied to the system to obtain the desired output. i.e., it is required to determine the PMSM wave currents $\left(i_{d}, i_{q}\right)$ and 
Voltages $\left(\mathrm{u}_{\mathrm{d}}, \mathrm{u}_{\mathrm{q}}\right)$ in order to obtain the desired output speed $(\omega)$.

Due to the nonlinearity and the complexity of the motor model, it is needed to decouple the system into two subsystems that are the electrical and the mechanical systems that shown in Fig.2.

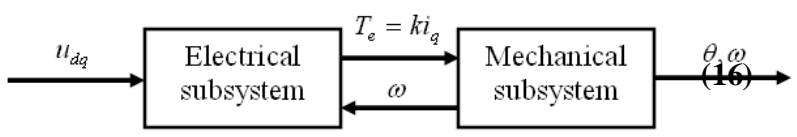

Fig.2: Decomposition into Mechanical and Electrical subsystems.

\subsection{Model of Electrical Subsystem}

$$
\begin{aligned}
& {\left[\begin{array}{l}
\dot{i}_{d} \\
\dot{i}_{u}
\end{array}\right]=\left[\begin{array}{l}
\dot{x}_{1} \\
\dot{x}_{2}
\end{array}\right]=\left[\begin{array}{cc}
-\frac{R}{L} & P x_{3} \\
-p x_{3} & -\frac{R}{L}
\end{array}\right]\left[\begin{array}{l}
x_{1} \\
x_{2}
\end{array}\right]+\left[\begin{array}{cc}
\frac{1}{L} & 0 \\
0 & \frac{1}{L}
\end{array}\right]\left[\begin{array}{l}
u_{d} \\
u_{q}
\end{array}\right]} \\
& T_{c}=\left[\begin{array}{ll}
0 & K
\end{array}\right]\left[\begin{array}{l}
x_{1} \\
x_{2}
\end{array}\right]
\end{aligned}
$$

In the Electrical system, the object is to produce output currents for driving the mechanical parts of the motor by adjusting the input voltages waveforms with knowing motor velocity.

\subsection{Model of Mechanical Subsystem}

In the Mechanical system, from the controlled input currents the motor rotates till reaching the desired velocity.

$$
\dot{\omega}=\dot{x}_{3}=\left[-\frac{\mu}{J}\right] x_{3}+\left[\frac{K}{J}\right] x_{2}-\left[\frac{1}{J}\right] T_{l}
$$

\section{DECOUPLED FUZZY SLIDING MODE CONTROL DESIGN}

The main idea behind the decoupled strategy is to decouple a nonlinear system appearing in the form of (6) into two subsystems as Electrical and Mechanical in the form of $(10,11)$. The Electrical subsystem is chosen as a(11) primary target while the Mechanical subsystem is used as a secondary target.

However, the selection of the primary and the secondary subsystems is problem dependent. Here, the control objective is to devise a control strategy that would move the states of both subsystems towards their sliding surfaces $S=0$. The Electrical subsystem involves knowledge from Mechanical subsystem, and the Mechanical subsystem is driven from the Electrical subsystem.

The electrical subsystem has three sub-controllers with three sliding surfaces $S_{1}, S_{2}$ and $S_{3}$ due to the three phases of the motor, i.e., SMC controller for each phase.

Let the sliding surfaces are defined as

$$
\begin{aligned}
& S_{1}=\dot{e}_{1}+c_{1} e_{1} \\
& S_{2}=\dot{e}_{2}+c_{2} e_{2} \\
& S_{3}=\dot{e}_{3}+c_{3} e_{3}
\end{aligned}
$$

Hence

$$
\begin{aligned}
& e_{1}=\left(i_{r a}-i_{a}\right) \\
& e_{2}=\left(i_{r b}-i_{b}\right) \\
& e_{3}=\left(i_{r c}-i_{c}\right)
\end{aligned}
$$

Where $c_{1}, c_{2}, c_{3}$ are the sliding surfaces constants for abc-axis $i_{r a}, i_{r b}$ and $i_{r c}$ are the references three phases currents. $i_{a}$, $i_{b}$ and $i_{c}$ are the actual three phases currents.

The Mechanical subsystem has sliding surface $S_{4}$ and is defined as

Hence $\quad S_{4}=\dot{e}_{4}+c_{4} e_{4}$

$$
e_{4}=\left(\omega_{r}-\omega\right)
$$

Where: $c_{4}$ is the sliding surface $S_{4}$ constant, $\omega_{\mathrm{r}}$ is the reference motor speed. $\omega$ is the actual motor speed.

The first step of fuzzy controller design is to know and define the linguistic values for all linguistic variables. Therefore, membership functions are needed for the linguistic variables. There are many ways to describe the membership functions. The important property of membership functions is the shape.

In the design of DFSMC controller, there are five steps:

First Step, The inputs of the controller will be an error value between reference input and feedback variable of the system as Eqs $(13,15)$. Then compute the sliding surface $S$ as in Eqs.(12,14).

Second Step, Fuzzification of input sliding surface by the input membership functions. The fuzzy(15) controller receives input information, then, applies it to the rule bases.

Third Step, Rule Inference, the fuzzy rule based system converts input information into output membership functions,

Forth Step, Defuzzification of output variable. There are many methods which can be used to obtain the constant output factor $\phi$ which defines the thickness of the boundary layer.

Fifth Step, Then the controller output signal u can be divided into an equivalent control input and a hitting control input, it has the following control law:

$$
u=u_{e q}-\operatorname{Msat}\left(\frac{S}{\phi}\right)
$$

Where: $\mathrm{M}$ is a positive constant, $u_{e q}$ is the equivalent.

$$
\operatorname{sat}\left(\frac{S}{\phi}\right)= \begin{cases}\frac{S}{\phi} & \text { if }\left|\frac{S}{\phi}\right| \leq 1 \\ \operatorname{sgn}\left(\frac{S}{\phi}\right) & \text { if }\left|\frac{S}{\phi}\right|<1\end{cases}
$$


IThe DFSMC block diagram can be shown in Fig.3 after determine the desired position and calculate the reference currents $i_{r a}, i_{r b}, i_{r c}$. DFSMC controller produces a control signal $u$, which drives the switching Inverter to produce the required voltage (v) for the motor. Observing the motor current position and angular velocity that to adapt the Sliding Mode for reaching the sliding surfaces.

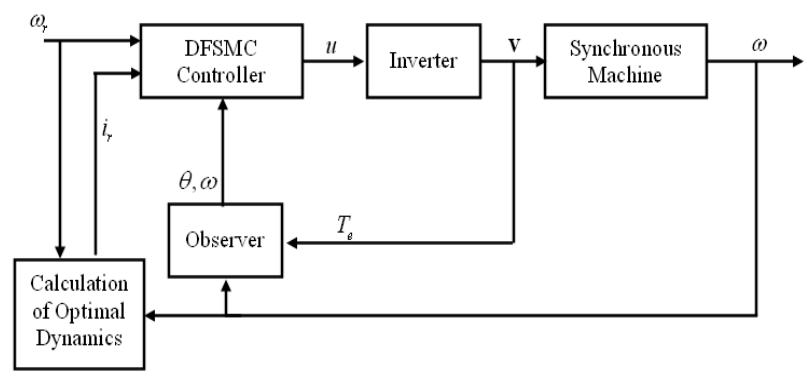

Fig.3: DFSMC controller Block diagram

\section{THE SIMULINK DFSMC CONTROLLER MODEL}

The DFSMC speed Controller is shown in Fig.4

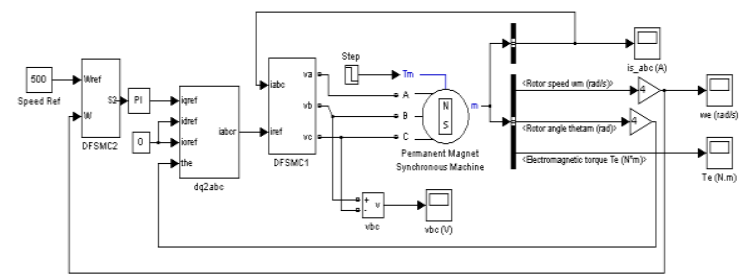

Fig.4: PMSM Speed Control Using DFSMC

The model contains PI block which is used to calculate the reference signal needed in dq-axis. A dq-abc-Transform block transfers the dq-currents to abc-currents format. The DFSMC2 starts to calculate the tracking error in speed $(14,15)$, hence produces the reference currents needed for the motor. DFSMC1 calculate the tracking error in currents (13-17), then produce output voltages to force the system towards the sliding surface first (Reaching), then DFSMCs try to keep the system slides on the switching surfaces till reaching the desired speed. The DFSMC1 contents shown in Fig.5.

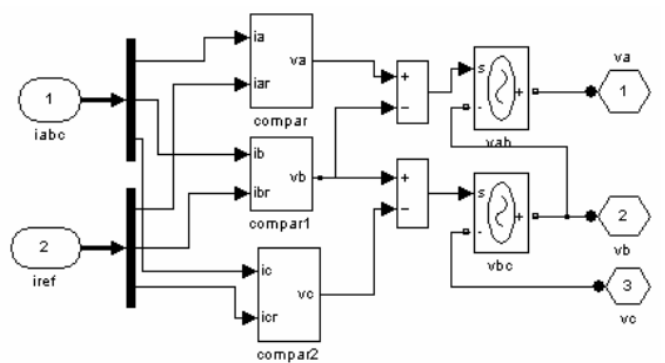

Fig.5: The DFSMC1 Controller

The contents of Compare, compare2 and compare 3 Shown in Fig.6, Fig.7 and Fig.8 respectively.

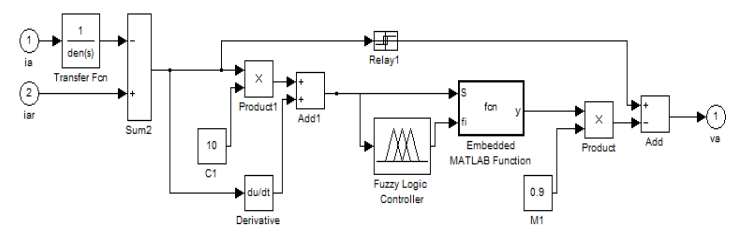

Fig.6: Compare Contents

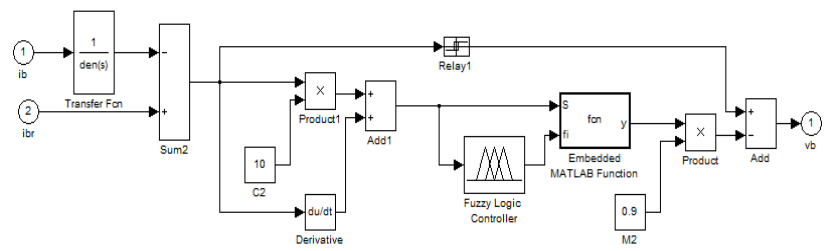

Fig.7 : Compare1 Contents

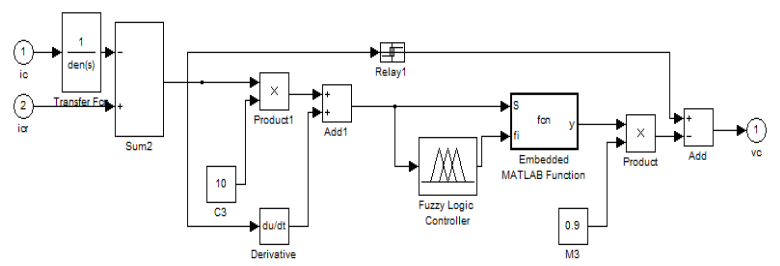

Fig.8: Compare2 Contents

The transfer function is a filter to reduce the harmonics with the measured current, and then satisfy the sliding surface $\left(S_{1}, S_{2}\right.$ and $\left.S_{3}\right)$ to obtain the control output. Selecting $c_{1}=c_{2}=c_{3}$ because of the contribution of each phase is similar to the others. The constants required for faster response. But not too large, otherwise the motor will suffer from torque ripples. When the DFSMC 1 moves toward the origin (the reference currents are near the actual currents) and with motor current ranges from nearly $+2.2 \mathrm{~A}$ to $-2.2 \mathrm{~A}$, larger constants $\mathrm{c}_{1}, \mathrm{c}_{2}, \mathrm{c}_{3}$ are required especially with small current differences. If these constants are too large then the error may change its sign and may induce torque ripples.

The DFSMC2 contents shown in Fig.9.

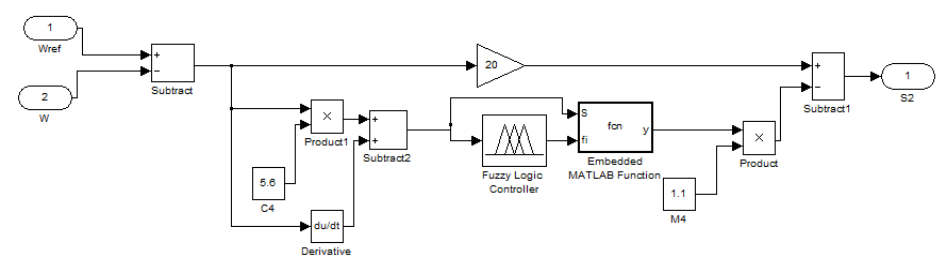

Fig.9: DFSMC2 Contents

For reaching the desired speed with faster response and without speed oscillations, it should select $\mathrm{c}_{4}$ not small than 1 for achieving faster response and not too large for reducing the speed oscillations.

The fuzzy rules are applied for the sliding surfaces $\left(S_{1}, S_{2}, S_{3}\right.$, $\mathrm{S}_{4}$ ) to obtain the thickness of the boundary layer.

\section{SIMULATION RESULTS}


Figure 10 summarizes the simulation results for a three-phase motor rated $1.1 \mathrm{~kW}, 3000 \mathrm{rpm}$. The load torque applied to the machine's shaft is originally set to its nominal value $(4 \mathrm{~N} . \mathrm{m})$ and steps down to $2 \mathrm{~N} . \mathrm{m}$ at $\mathrm{t}=0.04 \mathrm{~s}$. The desired speed 500 $\mathrm{rad} / \mathrm{sec}$.
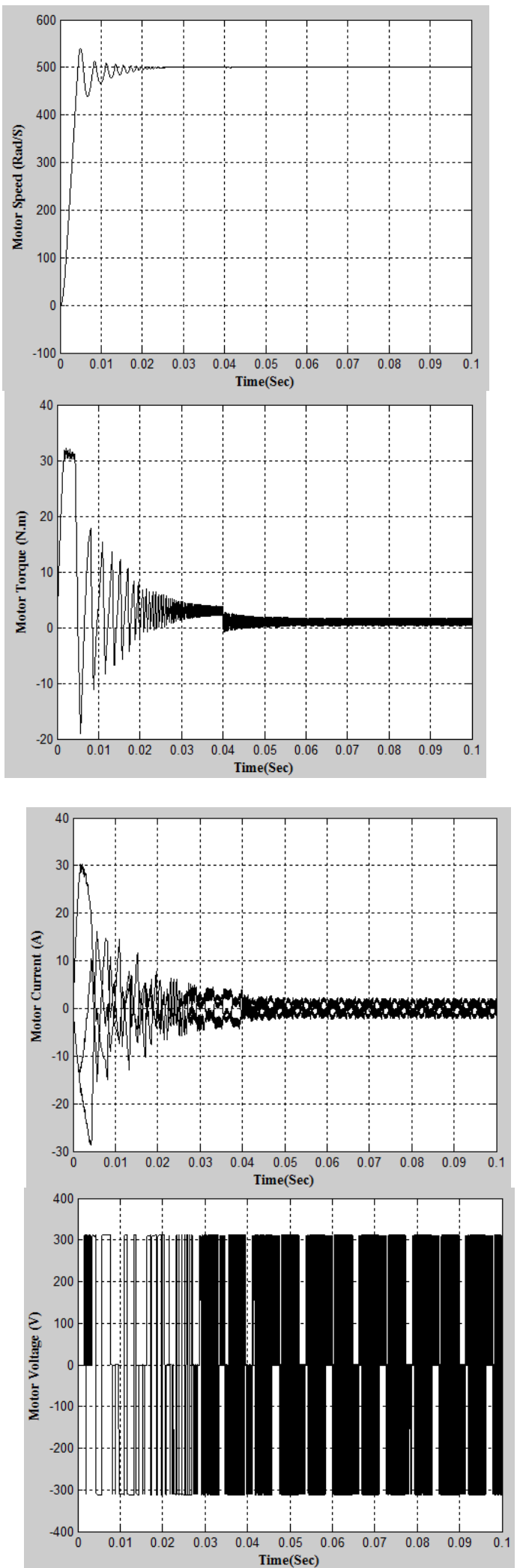

Fig.10: DFSMC Controller Simulation Curves of PMSM
Fig.10 shows the PMSM speed, torque, currents and voltage using DFSMC controller. These results can be summarized in the following:

- The reference speed is $500 \mathrm{rad} / \mathrm{sec}$, the motor speed stabilized at $0.025 \mathrm{sec}$. Table 1 summarizes the results

- The torque stabilized at the nominal torque 4 N.m till $0.04 \mathrm{sec}$. The motor torque drops to -0.77 N.m due to the torque reducing, then DFSMC controller tries to overcome the sudden torque changes and stabilize the torque at $1.8 \mathrm{~N} . \mathrm{m}$.

- The motor starts with high current $30.1 \mathrm{~A}$, and then the currents reduced and stabilized in sinusoidal waveform with max. $4.5 \mathrm{~A}$. When the load torque is reduced to $1.8 \mathrm{~N} . \mathrm{m}$ at $0.04 \mathrm{sec}$, all currents are reduced to max. $2.16 \mathrm{~A}$

- Chopping the motor voltage to achieve the required currents for adjusting the speed.

Table 1 Speed Results Using SMC

\begin{tabular}{|cc|}
\hline Time of reaching & $0.025 \mathrm{sec}$ \\
\hline Max. Speed deviation & $500.05 \mathrm{Rad} / \mathrm{sec}$ \\
\hline Min. Speed deviation & $499.93 \mathrm{Rad} / \mathrm{sec}$ \\
\hline Speed oscillations & Approx. $\pm 0.014 \%$ \\
\hline
\end{tabular}

\section{COMPARATIVE ANALYSIS}

Another technique can be used to control the speed of the PMSM [21-23]. PWM (Pulse Width Modulation) inverter is used to control the waveforms of currents and voltages that will be applied to the motor. The PWM controller shown in Fig.11

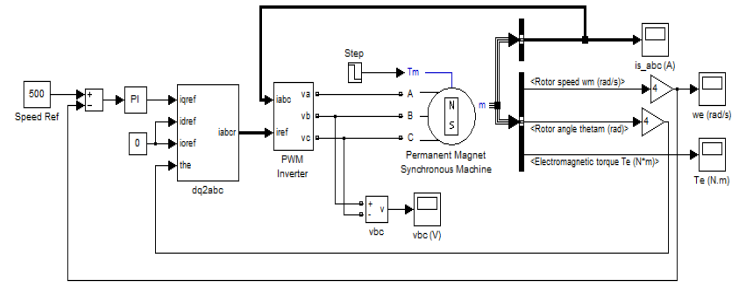

Fig.11: PMSM Speed Control Using PWM

Figure 12 summarizes the simulation results for PWM controller with the same motor parameters. 

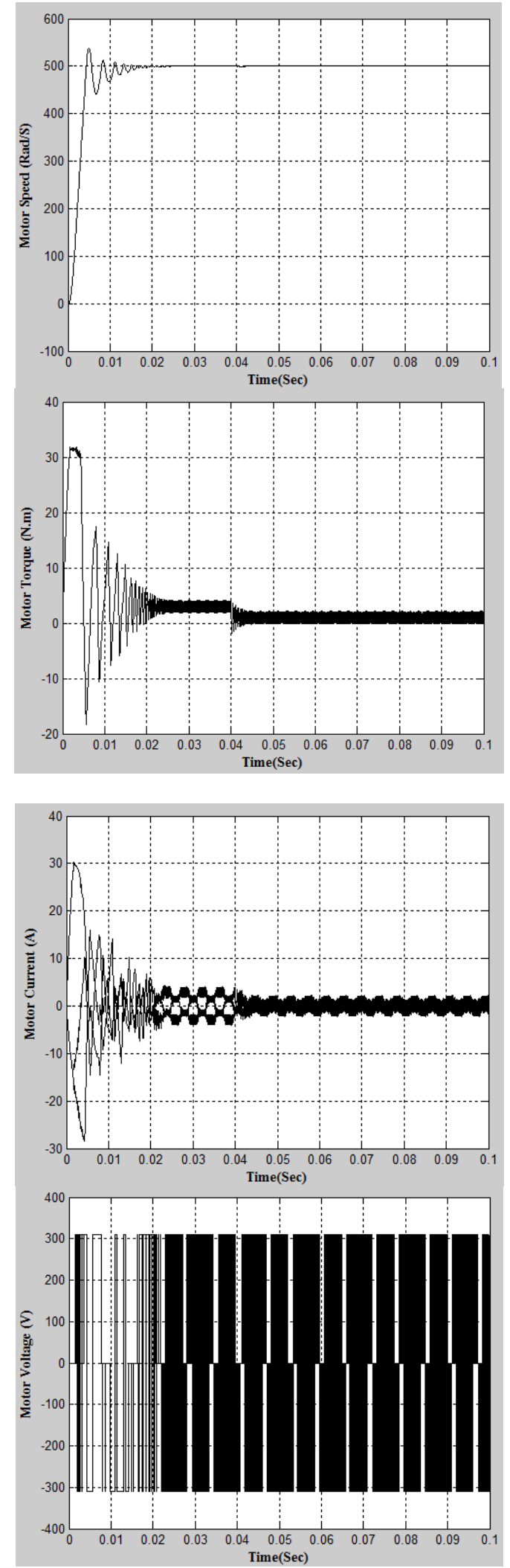

Fig.12: PWM Controller Simulation Curves of PMSM

Fig.12 shows the PMSM speed, torque, currents and voltage using PWM controller. These results can be summarized in the following:

- The reference speed is $500 \mathrm{rad} / \mathrm{sec}$, the motor speed stabilized at $0.024 \mathrm{sec}$. Table 2 shows the results
Table 2 Speed Results Using PWM

\begin{tabular}{|cc|}
\hline Time of reaching & $0.024 \mathrm{sec}$ \\
\hline Max. Speed deviation & $500.15 \mathrm{Rad} / \mathrm{sec}$ \\
\hline Min. Speed deviation (steady & $499.8 \mathrm{Rad} / \mathrm{sec}$ \\
\hline Speed oscillations (steady & Approx. $\pm 0.040 \%$ \\
\hline
\end{tabular}

From Table 1 and Table 2, it is noted that DFSMC controller reaches the motor speed close to the PWM controller. Also Fig.13 shows the speed oscillations for a sample time for both controllers at steady state which concluded that the speed oscillations in DFSMC controller is less than in PWM controller.
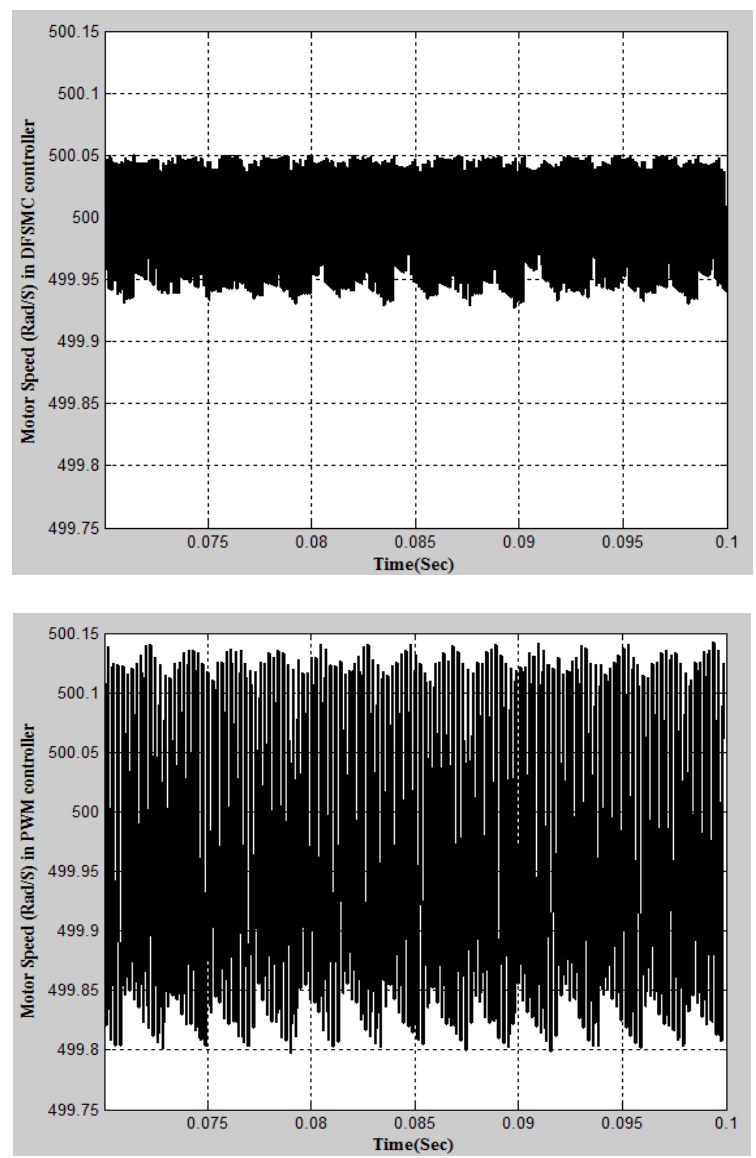

Fig.13: Steady State Sample Period Speed of PMSM

The motor torque drops to -1.789 N.m at $0.04 \mathrm{sec}$ due to the torque reducing. The compared results shown in Table 3

Table 3 Compared Torque Results

\begin{tabular}{|ccc|}
\hline Torque & PWM & DFSMC \\
\hline Torque & -0.1491 to 2.123 & 0.1873 to 1.81 \\
Range & N.m & N.m \\
& & \\
\hline Sudden & -1.789 N.m & -0.7768 N.m \\
Torque Drop & & \\
\hline
\end{tabular}


The torque in PWM controller switching between positive and negative torques which means high torque ripples then in DFSMC controller.

- The three phase currents have maximum current $2.2 \mathrm{~A}$ at steady state in PWM controller, which is the same at DFSMC controller.

- The voltage is chopped achieve the required motor currents and speed.

\section{CONCLUSION}

This paper presents the design of control system for controlling the speed of Permanent Magnet Synchronous Motor using the decoupled fuzzy sliding mode control algorithm. The decoupled technique divides the system into Electrical and Mechanical subsystems which can be separately modeled and controlled. The proposed controller assures its validity and effectiveness against the regular Pulse Width Modulation controller. Simulation results were presented for both controllers. DFSMC achieves less speed oscillation and low torque ripples compared with PWM.

\section{REFERENCES}

[1] Utkin, V.I. , IEEE Trans. Automat. Contr. 22 (1977) 212-222. Variable structure systems with sliding mode : A survey

[2] Chan, M.L., Tao , C.W., and Lee , T.T. Journal of the Franklin Institute 337 (2) (2000) 105-115. Sliding mode controller for linear systems with mismatched timevarying uncertainties

[3] Sha, D. , Bajic, V.B., and Yang, H. Simulat. Modell. Pract. Theory 9 (6) (2002) 365-385. New model and sliding-mode control of hydraulic elevator velocity tracking system

[4] Boiko , I. and Fridman, L. IEEE Transactions on Automatic Control, 50 ( 9) (2005) 1442-1446. Analysis of Chattering in Continuous Sliding-Mode Controllers

[5] Yunjun $\mathrm{Xu}$, IEEE Transactions on control Systems Technology, 16 (6) (2008) 1352-1359. Chattering Free Robust Control for Nonlinear Systems

[6] Yorgancioglu, F. , and Komurcugil, H. Expert Systems with Applications 37 (2010) 6764-6774. Decoupled sliding-mode controller based on time-varying sliding surfaces for fourth-order systems.

[7] Tai-Zu Wua,_, Jinn-Der Wangb, Yau-Tarng Juang . Journal of the Franklin Institute 344 (2007) 1006-1020. Decoupled integral variable structure control for MIMO systems.

[8] Yaodong Pan, Krishna Dev Kumar Guangjun and Katsuhisa Furuta. IEEE Transactions on control Automatic Control, 54 (8) (2009) 1981-1986. Design of Variable Structure Control System With Nonlinear TimeVarying Sliding Sector.

[9] Jian-Bo Cao and Bing-Gang Cao. IEEE Transactions on Power Electronics, 24 (10) (2009) 2368-2378. FuzzyLogic-Based Sliding-Mode Controller Design for Position-Sensorless Electric Vehicle.

[10] Lon-Chen Hung, Hung-Yuan Chung . International Journal of Approximate Reasoning 46 (2007) 74-97.
Decoupled sliding-mode with fuzzy-neural network controller for nonlinear systems.

[11] Hachemi Glaoui, Abdeldejbar Hazzab, Bousmaha Bouchiba, Ismaïl Khalil Bousserhane, and Pierre Sicard. International Journal on Electrical Engineering and Informatics 3 (1) (2011) 83-99. Fuzzy Sliding-Mode Control for a five drive web-winding System

[12] Q. P. Ha, Q. H. Nguyen, D. C. Rye and H. F. DurrantWhyte. IEEE Transactions on Industrial Electronics, 48 (1) . Fuzzy Sliding-Mode Controllers with Applications",

[13] Diantong Liu *, Jianqiang Yi , Dongbin Zhao and Wei Wang. Mechatronics 15 (2005) 505-522. Adaptive sliding mode fuzzy control for a two-dimensional overhead crane.

[14] Arash Hassanpour Isfahani, and Siavash Sadeghi. World Academy of Science, Engineering and Technology 45 (2008) 566-570. Design of a Permanent Magnet Synchronous Machine for the Hybrid Electric Vehicle.

[15] Mi , C. IEEE Transactions on Magn., 42 (2006) ,pp. 1861-1866. Analytical design of permanent-magnet traction-drive motors.

[16] Fujishima, Y., Vakao, S. , Kondo, M. , and Terauchi, N. IEEE Transactions on Applied Superconductivity, 14, (2004) pp. 1902-1905 . An optimal design of interior permanent magnet synchronous motor for the next generation commuter train.

[17] Magnussen, F. , Thelin, P. , and Sadarangani, C. in Proc. 20th Int. Electric Vehicle Symposium and Exposition, Long beach, California, USA, 15-19 Nov., (2003) 181191. Design of compact permanent magnet machines for a novel HEV propulsion system.

[18] Wu, S. , Song, L. , and Cui, S. IEEE Transactions on Magn., 43 (2007) 438-442. Study on improving the performance of permanent magnet wheel motor for the electric vehicle application.

[19] S. Vaez-Zadeh , and Ghasemi, A.R. Electric Power Systems Research, 74 (2005) 307- 313. Design Optimization of Permanent magnet Synchronous Motors for High Torque Capability and Low Magnet Volume.

[20] Javad Rezaie, Mehdi Gholami, Reza Firouzi, Tohid Alizadeh, and Karim Salashoor . Proceedings of the World Congress on Engineering and Computer Science 2007, WCECS 2007, October 24-26, 2007, San Francisco, USA, ISBN:978-988-98671-6-4. Interior Permanent Magnet Synchronous Motor (IPMSM) Adaptive Genetic Parameter Estimation.

[21] Zhang, W. , and Zhan, W. Proceedings of WCECS 2008 , October 22-24, 2008, San Francisco, USA, ISBN:978 988-98671-0-2. Sensitivity Analysis of Motor PWM Control.

[22] Dewangan, A. , Charkraborty , N., Shukla, S. and Yadu, V. International Journal of Engineering Trends and Technology, 3 (2012), 110-112. PWM based Automatic Closed Loop Speed Control of DC Motor

[23] Arulmozhiyal , R. and Baskaran, K. International Journal of Computer and Electrical Engineering, 1 (2009) 98103. Space Vector Pulse Width Modulation Based Speed Control of Induction Motor using Fuzzy PI Controller. 